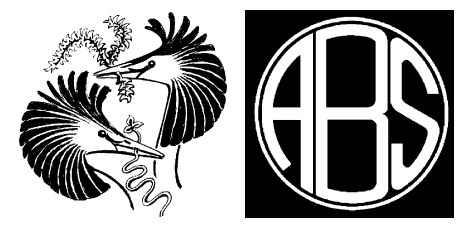

\title{
Dog-logic: inferential reasoning in a two-way choice task and its restricted use
}

\author{
ÁGNES ERDÖHEGYI*, JÓZSEF TOPÁL†, ZSÓFIA VIRÁNYI* \& \\ ÁDÁM MIKLÓSI* \\ *Department of Ethology, Eötvös Loránd University, Budapest, Hungary \\ $\dagger$ Institute of Psychology, Hungarian Academy of Sciences, Budapest, Hungary \\ (Received 2 August 2005; initial acceptance 7 March 2007; \\ final acceptance 9 March 2007; published online $\mathbf{\square} \mathbf{\square}$; MS. number: 8642R4)
}

\begin{abstract}
Three experiments were designed to test whether adult pet dogs are able to show inferential reasoning when searching for their toy in a series of two-way choice tasks. The experimenter placed a toy under one of two identical containers and then provided some information by manipulating the covers: either both containers were lifted or just the empty or baited one. There were other trials when the experimenter not only revealed the corresponding container but manipulated also the other one without showing its content. In the second experiment the same conditions were used except that the content of the containers was revealed by strings without any human manipulation. Results of the two studies show that dogs are able to use inferential reasoning by exclusion (i.e. they can find the hidden toy if they have seen where the toy was missing). However, dogs were able to solve the reasoning task only when they could not rely on social-communicative cues (directional gesture and gaze cues) or could not use any other simple discriminative stimuli (movement of a container) for making decisions. This suggests that dogs are often prevented from showing reasoning abilities by pre-existing biases for social or movement cues. Results of the third experiment also support the primary importance of social cueing because in another object-choice task, individuals preferred to choose the 'socially marked' container (touching, gaze alternation) to the remotely moved one when they had no visual information about the location of the toy.
\end{abstract}

(c) 2007 The Association for the Study of Animal Behaviour. Published by Elsevier Ltd. All rights reserved.

Keywords: cognition; decision by exclusion; deduction; dog; reasoning

Despite the wide range of studies based on human and animal cognition published in the past decades, the evolutionary emergence of reasoning abilities including different types of logical reasoning in humans continues to be a puzzle for cognitive science (Wright 2001). The fundamental question is whether an observed problemsolving behaviour is a result of gradual development in performance due to learning processes or is a sudden solution emerging from mental reorganization of problem elements (i.e. insight: Köhler 1925). In distinguishing between learning and reasoning, many assume that even though the more parsimonious learning processes can

Correspondence: Á. Erdöhegyi, Department of Ethology, Eötvös Loránd University, H-1117 Budapest, Pázmány P. 1/c, Hungary (email: agnes. erdohegyi@gmail.com). J. Topál is at the Institute of Psychology, Hungarian Academy of Sciences, H-1117 Budapest, Pázmány P. s. 1/c, Hungary. never be fully excluded (Heyes 1993), reasoning can be presumed if the subject shows an 'insightful solution' immediately (i.e. shows adequate behaviour without explicit training) in the first few trials (Premack 1995).

To test for reasoning one may present a problem and provide sufficient but indirect information to solve it. One way to study the reasoning abilities is the Piagetian object permanence paradigm. In the invisible displacement task, no direct cues specify that the desired reward has moved behind the screen (i.e. the subject does not witness the reward being dropped). The subject must infer, upon seeing the empty container, that the object has been transferred. Previous studies with several species showed that some primates (e.g. orang-utans, Pongo pygmaeus: de Blois et al. 1998; orang-utans: Call 2001; chimpanzees, Pan troglodytes: Wood et al. 1980; cottontop tamarins, Saguinus oedipus: Neiworth et al. 2003), magpies, Pica pica (Pollok et al. 2000), grey parrots, Psittacus erithacus (Pepperberg et al. 1997) and dogs, Canis familiaris 
(Gagnon \& Doré 1992, 1993) were able to solve the single invisible displacement tasks. However, it is doubtful whether dogs really used inferential reasoning to find the reward or whether they solve the problem by using local rules (Watson et al. 2001; Collier-Baker et al. 2004, 2006).

A simpler way to study inferential reasoning by exclusion in animal species is two-way choice tasks. The paradigmatic element of this approach presumes, as it is in the object permanence task, that subjects are exposed to either direct (witness the hiding) or indirect information (e.g. the sight of an empty box).

In this case apes are known to be skilful at making inferences by exclusion about the location of hidden food. Premack \& Premack (1994) reported that when chimpanzees can see a human putting two types of fruit in two boxes (one in each) and later are allowed to witness the same person eating one of the fruits, they prefer to choose the box containing the other type of fruit.

Call \& Carpenter (2001) reported that apes (chimpanzees and orang-utans) were correctly able to infer the location of food in one of several tubes based on the contents of the first tube(s) they looked into. Subjects were presented with a finding game in which food or stickers were hidden in one of two or three tubes. They varied whether subjects saw the baiting of the tubes, whether subjects could see through the tubes, and whether there was a delay between baiting and presentation of the tubes to subjects. They measured not only whether subjects chose the correct tube but also whether they spontaneously looked into one or more of the tubes before choosing one. Most subjects appropriately looked into the tubes before choosing one, but they did this more often when they had not seen the baiting, than when they had seen the baiting and were able to infer the location of the reward.

More recently, Call (2004) reported a series of two-way choice tasks using direct and/or indirect informing cues either in visual or in auditory modality in apes. His subjects preferred to choose the baited container even when both containers were manipulated but only the empty one was either shown or shaken, and concluded that their performance was based on inferential reasoning by exclusion rather than on associative learning.

In addition to the numerous ape studies (see Tomasello 2000 for review), experimental observations on other species are important in order to broaden our understanding of evolutionary emergence of reasoning abilities. We may assume that the domestic dog is a promising subject because its ancestor was a cooperative hunting carnivore, which could be a determining factor in the evolution of sophisticated constructional skills (Gibson 1990). Domestic dogs are evolutionary distant relatives of humans, but have been subjected to very similar ('human-like') adaptational demands in the past 10000 years. This could account for the dog's sophisticated social cognition (see Hare \& Tomasello 2005 and Miklósi \& Topál 2005 for a recent discussion). Dogs are not only able to make inferences about the communicative meaning of human gestural cues as in indicating the location of reward (e.g. Soproni et al. 2001) but they seem to understand the communicative cues in complex 'triadic' situations where a human alternately interacts either with them or with another human as well (Virányi et al. 2004).

On the other hand, some results from understanding means-end connection show that dogs are not able to process sophisticated forms of physical causality (Osthaus et al. 2005) and lend support to the speculations that this 'inability' is the indirect consequence of the domestication process (Frank 1980; Hare et al. 2002; but see Miklósi et al. 2004 for review).

Other studies (Szetei et al. 2003; Bräuer et al. 2006) have shown that in particular situations dogs, unlike apes, are more willing to rely on human communicative and behavioural cues to find a reward than on perceptual cues of the physical world or on causal cues. For example, dogs chose the empty container when they received direct perceptual information about the place of the food (subjects were allowed to sniff both containers) and a contradictory communicative cue (human pointed at the empty container; Szetei et al. 2003).

Some studies based on object permanence paradigm suggest that the ability to understand causal relationships as well as responsiveness to social cues influence the performance of dogs in simple hide-and-search tasks (Watson et al. 2001; Topál et al. 2006). This means that the inferential reasoning of dogs in a search task may be affected by pre-existing biases for social cues. In general, the two-way object-choice task does not separate the perceptual information from the communicative cues because showing the empty container to the subject involves both kinds of information (manipulating the object and revealing its content). In the present experiment, dogs were provided with direct and indirect visual cues about the location of their favourite toy, a ball, to see whether their choice behaviour could be interpreted in terms of inferential reasoning. In the first experiment, visual cues (sight of the toy or sight of the empty container) and the attention-getting/social-influencing effect of human manipulation (touching, moving and looking at the container) were combined independently in the different experimental conditions.

\section{EXPERIMENT 1}

\section{Methods}

\section{Subjects}

Forty-two adult pet dogs and their owners were recruited on voluntary basis. The only criterion for selection was that the dogs had to be highly motivated to play with a toy.

Three dogs were rejected during the first few trials because they lost interest in finding and retrieving the ball and an additional four subjects were excluded from the final analyses due to significant side preference (i.e. regardless of the actual place of the toy they approached the same container at least 10 times out of the 12 test trials, binomial test for side bias: $P<0.04)$.

The remaining 35 dogs (23 males, 12 females; mean age $\pm \mathrm{SD}=4 \pm 2.28$ years) included 15 different recognized breeds (7 Parson Russel Terriers, 5 German 
Shepherd Dogs, 2 Malinois, 2 Labrador Retrievers, 2 Golden Retrievers, 2 Rotweilers, 2 German Pointers, 1 Miniature Pinscher, 1 Springer Spaniel, 1 Jack Russel Terrier, 1 Border Collie, 1 Pumi, 1 Dobermann, 1 Argentine Dog, 1 Mudi) and five mongrels.

All owners were unaware of the hypotheses and goals of the study.

\section{Procedure}

Experimental arrangement. The experiments were recorded in a room $(5 \times 2.5 \mathrm{~m})$ where two identical containers (turned upside down) were placed $1.5 \mathrm{~m}$ apart to hide the toy. Containers were composed of an outer part (a brown plastic flowerpot, $22 \mathrm{~cm}$ in diameter) and an inner one, a smaller brown plastic flowerpot $(20 \mathrm{~cm}$ in diameter). These were telescoped so that they could be lifted together or one at a time by means of two strings (Fig. 1).

Pretest trials. The owner made the dog stand at a predetermined point in the room. The experimenter nicknamed the dog and placed the two containers equidistant from the dog $(2 \mathrm{~m})$. Next she caught the dog's attention with the toy in her hand and then placed it under one of the containers while she alternated her gaze three times between the dog and the baited container. After baiting, she lifted up the baited container to about $40 \mathrm{~cm}$ to reveal the ball under the container for $3 \mathrm{~s}$; meanwhile, she alternated her gaze three times between the dog and the manipulated container. Finally, the experimenter replaced the container, took up her initial position, turned her back to the dog and the dog was allowed to choose. If the dog chose the baited container, it was allowed to play with the toy for a few seconds. The trial was repeated once more, but instead by hiding the toy under the other container. In trials 3 and 4 , the same procedure was repeated with the exception that after the baiting, the experimenter lifted only the outer part of container (in the same way as described above). All dogs (except those who were excluded due to motivational problems: see Subjects) met the criterion of 3 or 4 correct choices.

Test trials. Each trial consisted of the following three phases.

(1) Baiting: Having been called and shown the ball, the dog was prevented from witnessing the concealment by a green plastic barrier. The experimenter manipulated the left and then the right container and left the ball under one of them. Then she took up her initial position

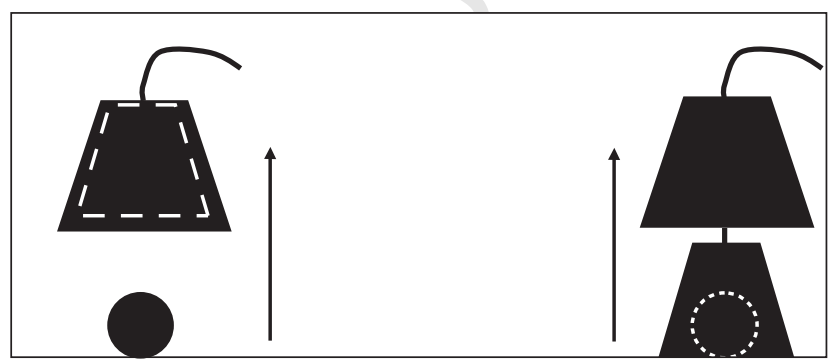

Figure 1. Possible ways of lifting the containers. between the containers and the visual barrier was removed.

(2) Providing information: The experimenter called the dog and administered one of the six following conditions.

(a) Both: The experimenter showed the content of both containers: first she stepped to the container on her left hand side and lifted it up (both the inner and the outer parts) revealing its content for $3 \mathrm{~s}$. In parallel, she alternated her gaze three times between the dog and the manipulated container. Next she went to the other container on her right and repeated the same procedure.

(b) Baited only: The experimenter showed only the content of the baited container. She went to the baited container and lifted it up to reveal its content for $3 \mathrm{~s}$ and alternated her gaze between the dog and the baited container. She did not approach and touch the empty container.

(c) Balanced baited: The experimenter showed only the content of the baited container (revealing its content for $3 \mathrm{~s}$ and alternating her gaze between the dog and the baited container). She also lifted the outer part of the empty container and made gaze alternations between the dog and the empty container.

(d) Empty only: The experimenter showed the content of the empty container. She went to the empty container and showed its content, making gaze alternations between the dog and the container, but she did not approach and touch the baited container.

(e) Balanced empty: The experimenter showed only the content of the empty container but lifted also the outer part of the baited container accompanied by gaze alternations between the dog and the container.

(f) Control: The experimenter manipulated both containers while she alternated her gaze three times between the dog and the hiding places. She did not provide information about the content of the containers because she lifted only their outer parts.

(3) Selecting a container: After these demonstrations the experimenter returned to her starting position and turned her back to the dog, and the owner released the dog to allow it to make a choice. If the dog chose the baited container, it was allowed to play with the ball for a few seconds.

Each dog received two trials per condition (one left and one right baiting) in a single session (12 trials in all). The order of the conditions and reward positions (left versus right) were predetermined and semirandom with the restriction that the ball did not appear more than two times in succession on the same side.

The whole session was videotaped and the choice behaviour of the subject was analysed later.

\section{Data collection and analysis}

A container was regarded as chosen by the dog if the dog turned it over, or touched it with its paw/muzzle or at least approached it (its paw/muzzle was closer than $10 \mathrm{~cm}$ to the container). To assess interobserver agreement a second person blind to the conditions scored a randomly selected sample of $20 \%$. Cohen's kappa value was $1(100 \%$ agreement), showing an extremely high level of reliability. 


\section{Analysing the overall performance}

The number of correct choices $(0,1$ or 2$)$ was compared between the different testing conditions using nonparametric statistical methods (Friedman ANOVA, Wilcoxon two-sample test and Wilcoxon signed-ranks test with false discovery rate correction [FDRbl adjustment, see Benjamini et al. 2001]) at the group level.

\section{Testing the relevance of the choice rules for the description of the dogs' performance}

In principle, subjects could choose between the two possible target places on the basis of many simultaneous decision-making processes which can be formulated into a series of 'choice rules'. These local rules (Natale et al. 1986) may be effective in helping subjects to recover the target object. Therefore, we formulated seven simple rules that could control the behaviour of the dog during the tests.

The detailed descriptions of the rules are as follows (see also Table 1).

Deductive inferring: 'Go to the other container if you have seen one being empty!' Correct choice could be made on the basis of this rule in those conditions where the dog had visual access to the content of the empty container (Both, Empty only, Balanced empty).

Using direct visual information: 'Choose the container under which you have seen the ball!' Dogs could rely on this rule when the experimenter showed the content of the baited container (Both, Baited only and Balanced baited conditions).

Preferring the moving container: 'Visit the container which was moved!' Dogs could rely on this rule when only one container was lifted up by the experimenter to reveal its content (Baited only and Empty conditions).

Preferring the container moved last: 'Visit the container which was last moved!' In those conditions when the experimenter moved both containers (Both, Balanced baited, Balanced empty and Control conditions), the order of the moving container could cause a bias in dogs' choice behaviour (having an increased attractiveness to the container moved last).

Preferring the container manipulated exclusively by the human: 'Visit the container which was manipulated by the experimenter!' In those conditions when only one of the containers had been manipulated (Baited only, Empty only), dogs' choice behaviour could be based on the human manipulation which may have increased the attractiveness of that container.

Preferring the container manipulated last by the human: 'Go to the container which was last manipulated by the experimenter!' In those conditions, when the experimenter manipulated both containers (Both, Balanced baited, Balanced only, Control), the sequence of human cueing could cause a bias in dogs' choice behaviour (having a stronger aftermath of human cueing in case of the last manipulated container).

Preferring the container rewarded previously: 'Go to the container under which the toy was hidden during the previous trial!' The sight of the reward under one of the containers (and getting the toy from under it) could cause the selection of the same container independently of the informing cues in the subsequent trial. Although the willingness to perform such perseverative actions often leads to erroneous choice behaviour (Watson et al. 2001),

Table 1. Relevance of the predetermined choice rules on the dogs' $(N=35)$ performance in the different experimental conditions in Experiments 1 and 2

\begin{tabular}{|c|c|c|c|c|c|c|c|c|}
\hline \multirow[b]{2}{*}{ Conditions } & \multirow[b]{2}{*}{ Experiment } & \multicolumn{7}{|c|}{ Descriptive name of the choice rules } \\
\hline & & $\begin{array}{c}\text { Deductive } \\
\text { inferring }\end{array}$ & $\begin{array}{l}\text { Using direct } \\
\text { visual } \\
\text { information }\end{array}$ & $\begin{array}{l}\text { Preferring } \\
\text { the moving } \\
\text { container }\end{array}$ & $\begin{array}{l}\text { Preferring } \\
\text { the } \\
\text { container } \\
\text { moved last }\end{array}$ & $\begin{array}{l}\text { Preferring } \\
\text { the } \\
\text { container } \\
\text { manipulated } \\
\text { by the } \\
\text { human }\end{array}$ & $\begin{array}{c}\text { Preferring } \\
\text { the container } \\
\text { manipulated } \\
\text { last }\end{array}$ & $\begin{array}{l}\text { Preferring the } \\
\text { container } \\
\text { rewarded } \\
\text { previously }\end{array}$ \\
\hline \multirow[t]{2}{*}{ Both } & 1 & $34-1-0$ ** & $34-1-0^{* *}$ & - & $1-34-0$, NS & - & $1-34-0$, NS & $3-7-25^{* *}$ \\
\hline & 2 & $27-3-0^{* *}$ & $27-3-0^{* *}$ & - & $1-28-1$, NS & - & - & $3-11-16^{* *}$ \\
\hline \multirow[t]{2}{*}{ Baited only } & 1 & - & $34-1-0^{* *}$ & $34-1-0 * *$ & - & $34-1-0$ ** & - & $6-12-17$, NS \\
\hline & 2 & - & $29-1-0-* *$ & $22-8-0^{* *}$ & - & - & - & $1-8-21^{* *}$ \\
\hline \multirow{4}{*}{$\begin{array}{l}\text { Balanced } \\
\text { baited } \\
\text { Empty only }\end{array}$} & 1 & - & $28-7-0^{* *}$ & - & 4-28-3, NS & - & $4-28-3$, NS & $3-14-18^{* *}$ \\
\hline & 2 & - & $25-4-1$ ** & - & $4-24-2$, NS & - & - & $3-10-17^{* *}$ \\
\hline & 1 & $2-14-19^{* *}$ & - & $19-14-2^{* *}$ & - & $19-14-2^{\star *}$ & - & $11-18-6$, NS \\
\hline & 2 & $1-15-14$ ** & - & $14-13-3^{*}$ & - & - & - & $6-17-7$, NS \\
\hline \multirow{4}{*}{$\begin{array}{l}\text { Balanced } \\
\text { empty } \\
\text { Control }\end{array}$} & 1 & $16-19-0^{* *}$ & - & - & $9-16-10$, NS & - & $9-16-10$, NS & $7-22-7$, NS \\
\hline & 2 & $11-18-1$ ** & - & - & $3-13-14^{*}$ & - & - & 5-14-11, NS \\
\hline & 1 & - & - & - & $9-16-10, \mathrm{NS}$ & - & $9-16-10$, NS & $10-16-9, \mathrm{NS}$ \\
\hline & 2 & - & - & - & $2-20-8$, NS & - & - & $2-16-12^{*}$ \\
\hline
\end{tabular}

The first numeral labels the number of dogs showing full correspondence with the rule (two corresponding choices), the middle numeral labels the number of dogs showing no preferential use of the rule (one corresponding choice) and the third numeral labels the number of subjects ignoring the rule (zero corresponding choice). The levels of significance are indicated by ${ }^{*}<0.05$ and ${ }^{* *} P<0.01$. Asterisks are bolded where dogs' choice behaviour corresponds with the rule significantly better than chance performance (Wilcoxon signed-ranks test with FDRbl adjustment, hypothetical median $=1$ ) and they are not highlighted where dogs show significant ignorance of the rule. 
in principle, the dog's behaviour can be influenced by this in each condition.

Importantly, these rules are not exclusive and in certain conditions, the rule on which the dog relies is indistinguishable (see Table 1). At the same time some of these choice rules can be nonrelevant in certain conditions (e.g. when the human informant does not show the content of the baited container, it is not possible to make a choice using direct visual information).

The explanatory value of each rule in each condition for each dog was defined as the number of trials $(0,1$ or 2$)$ in which the choice behaviour of the dog was in accordance with the choice resultant from the rule. In line with these subjects, they can be categorized into three main groups. Their choice behaviour corresponds with the rule in both trials (full correspondence) or only in one of the trials (no preferential use of the rule) or in neither of the trials (ignoring the rule). Using this categorization we have analysed whether subjects relied on any of the choice rules based upon their decision or showed explicit ignorance of that rule (Wilcoxon signed-ranks tests with FDRbl adjustment).

\section{Results}

\section{Comparing the number of correct choices between the testing conditions}

The performance of the dogs was strongly influenced by the types of the test trials $\left(\chi_{(5,35)}^{2}=110.74, P<0.0001\right.$, Fig. 2$)$.

The number of correct choices was at chance level in the Control condition $(T(-)=T(+)=85.5, N=35, P=1)$. The performance of the dogs was significantly above chance level when they were provided direct information about the location of the toy (Both, Baited and Balanced baited conditions: $T(-)=0, N=35, P<0.0001$ in each)

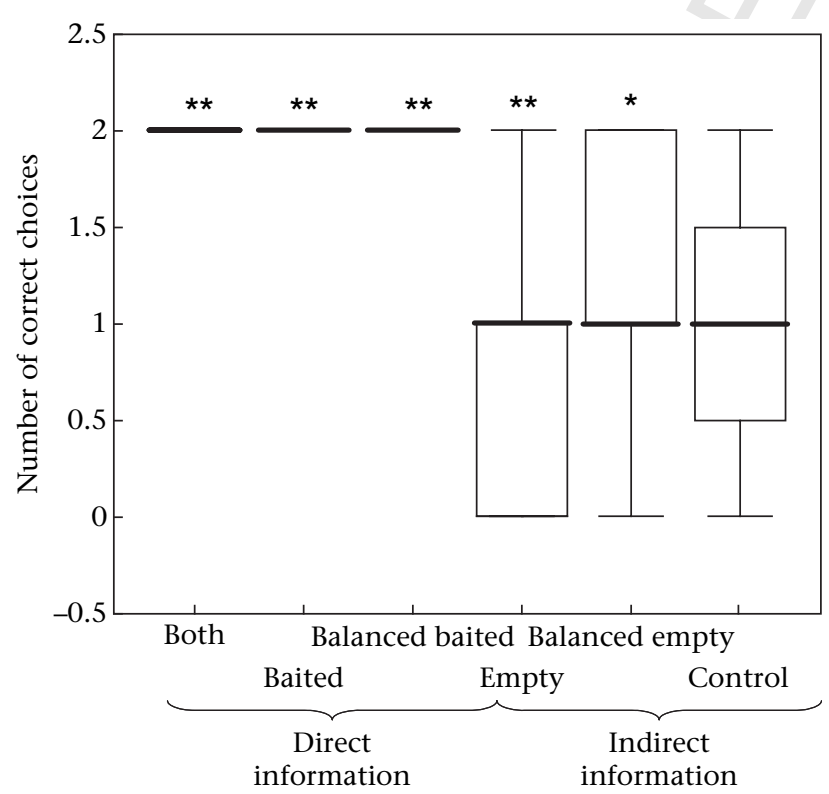

Figure 2. The number of correct choices in the different types of test trials in the 'social' task (Experiment 1): median, quartiles and extremes. Significant differences from control are indicated by ${ }^{*} P<0.05,{ }^{* *} P<0.01(N=35$, Wilcoxon signed-ranks tests with FDRbl adjustment). and also when they were shown the content of only the empty container, although both containers had been manipulated by the experimenter (Balanced empty: $T(-)=8.5, N=35, P<0.001)$. In these three conditions the number of correct choices was significantly above the performance level shown in the Control trials (Both condition: $T(-)=9.5, N=35, P<0.01$; Baited only condition: $T(-)=0, P<0.01$; Balanced baited condition: $T(-)=8.5, N=35, P<0.01$; Balanced empty condition: $T(-)=-26.0, N=35, P<0.05)$.

However, when the experimenter had manipulated only the empty container showing its content (Empty condition), the dogs chose the baited one below chance level $(T(+)=10.5, N=35, P<0.0001)$ and their performance was significantly lower compared with that shown in the Control condition $(T(+)=45.0, N=35, P<0.01)$.

\section{Testing the relevance of the choice rules for the description of the dogs' performance}

Table 1 shows the dogs' reliance on the predetermined choice rules in each condition and summarizes the results of the statistical analyses.

Comparisons of the observed choice behaviour in Both condition with the chance performance (no preferential use of the rule hypothetical median $=1$ ) have shown preferential use of the rules 'Deductive inferring' and 'Using direct visual information' $(T(-)=0, N=35, P<0.01$ for both) and significant ignorance of 'Preferring the container rewarded previously' $(T(+)=43.5, N=35, P<0.01)$. More importantly, however, in this condition the possible effects of 'Deductive inferring' and 'Using direct visual information' cannot be distinguished because both of them suggest the same choice (i.e. the baited container).

In the Baited only condition the dogs' behaviour corresponded significantly more strongly with the choices suggested by the 'Using direct visual information' rule, the 'Preferring the moving container' rule and the 'Preferring the human-manipulated container' rule (comparison with chance performance: $T(-)=0, N=35, P<0.01$ ).

In this case we could not decide whether dogs preferred to rely on direct visual information or on human cueing and on movement of the baited container because the aforementioned three rules had the same outcome (i.e. the experimenter manipulated and lifted only the baited container).

When the dogs' choices are compared with the chance level performance in the Balanced baited condition, their behaviour corresponded significantly with the 'Using direct visual information' rule $(T(-)=0, N=35$, $P<0.01)$ but not with those other choice rules which can be referred to as relevant in this condition. In addition, the rule 'Preferring the container rewarded previously' was significantly ignored by the dogs in this condition $(T(+)=33, N=35, P<0.01)$.

In those trials when the experimenter manipulated and revealed the content of only the empty container (Empty only condition) dogs seemingly did not use the 'Deductive inferring' rule (their choice behaviour fits this rule significantly less than what is expected by chance; $T(+)=22, N=35, P<0.01)$, while they show a significant 
willingness to make decisions as predicted by the rule 'Preferring the container manipulated by the human' and by the rule 'Preferring the moving container' $(T(-)=22, N=35, P<0.01$ in each). However, in contrast to the Empty only trials, in the Balanced empty condition (the human manipulated, touched and looked at both containers revealing only the content of the empty one), dogs' choice behaviour could be explained by the 'Deductive inferring' rule $(T(-)=0, N=35, P<0.01)$. However, they did not follow the other local rules ('Preferring the last manipulated container', 'Preferring the container moved last' and 'Preferring the previously rewarded container').

None of the predetermined local rules seemed to be relevant for explaining the dogs' choice behaviour in Control trials. That is, when the human informant manipulated equally both containers but did not give any relevant information about the location of the bait, dogs preferred neither of the choice rules (comparisons with chance performance: 'Preferring the last manipulated container' $T(-)=150, N=35, P=\mathrm{NS}$, 'Preferring the container moved last' $T(-)=150, N=35, P=\mathrm{NS}$ and 'Preferring the previously rewarded container' $T(-)=117$, $N=35, P=$ NS).

\section{Discussion}

These results show that, not surprisingly, dogs were able to solve the search-for-the-toy task when they had been informed about the location of the toy by direct visual cues (sight of the toy). However, in those conditions in which a human gave indirect visual cues (sight of the empty container) the dogs' performance was strongly influenced by the accompanying cues. When human manipulation was unbalanced (i.e. only the nonrewarded container was moved and marked by the human socially), dogs showed clear preference for the obviously empty container suggesting that these perceptual cues could supersede the reasoning abilities in dogs. In this case, reasoning has been seemingly counteracted by the rule suggesting preference for the moving container which was also manipulated communicatively (touching the container, gaze alternation between the container and the dog) by the human.

In contrast, when dogs could observe communicative cues (looking at and touching) and movement for both target places in a balanced fashion, they were able to choose the rewarded container even if only the content of the empty one had been shown. This suggests that dogs can still infer the location of their toy relying on the 'Deductive inferring' rule.

Unconscious cueing of the human participants or smell of the reward did not influence the dogs' choice behaviour as their performance was at chance in the control condition. Results suggest that dogs are able to perform simple inferential reasoning, but in fact they are often prevented from showing this competence by pre-existing biases for such salient cues as movement of the container and social marking of some hiding locations. However, in this experiment these two types of perceptual information could not be separated from each other and the relative importance of 'social marking' versus moving of the container could not be established.

\section{EXPERIMENT 2}

In a subsequent experiment, the effects of the movement of the container (without human communicative cues) were tested. In this case the containers were moved remotely by strings without any perceivable human manipulations. The purpose of this experiment was to investigate whether the performance of dogs in the 'inferential reasoning task' improves if the unbalanced human social-communicative cues are removed from the experimental procedure.

\section{Methods}

\section{Subjects}

By means of the same recruitment procedure and selection criteria as in Experiment 1, 36 dogs were entered on a voluntary basis by private owners. The only criterion for selection was that the dogs had to be highly motivated to play with a toy.

Two dogs were rejected during the first few trials when they lost interest in finding and retrieving the ball, an additional subject was excluded from the final analyses due to significant side preference (i.e. regardless of the actual place of the toy, she/he approached the same container at least 10 times out of the 12 test trials: binomial test for side bias: $P<0.06$ ), and three other dogs were rejected due to stressful behaviour when the containers were moved by strings.

The remaining 30 dogs (15 males, 15 females; mean age $\pm \mathrm{SD}=4.35 \pm 2.44$ years) included 14 different recognized breeds (3 German Shepherd Dogs, 2 Black Russian Terriers, 4 Vizslas, 4 Labrador Retrievers, 3 Malinois, 1 Jack Russel Terrier, 1 Border Collie, 1 Groenendael, 1 Argentinean Dogo, 1 Mudi, 1 Jagd Terrier, 1 Shcipperke, 1 Australian Shepherd, 1 Tibetan Terrier) and five mongrels.

All owners were unaware of the hypotheses and goals of the study.

\section{Procedure}

Experimental arrangement. Experimental observations were recorded in the same room as in Experiment 1. In this case, however, the experimenter could show the content of the containers without any direct manipulation by hand (they were moved remotely by thin nylon strings).

The same four containers that were used in Experiment 1 had individual strings attached to each container and were arranged in the same manner as they were in Experiment 1, but the strings ran out in an adjacent room from where movements of the containers could be controlled.

Two experimenters participated in the test. One (E1) stayed in the experimental room and managed the pretest trials and the baiting during the test trials. The other (E2) 
remained in the adjacent room and controlled the movement of the containers by the strings according to the test conditions.

Pretest trials. These were the same as in Experiment 1 except that the container that was chosen by the dog was lifted up by the strings, not by E1 (without any human manipulation).

Test trials. Each trial consisted of the following three phases:

(1) Baiting: see Experiment 1.

(2) Providing information: This was the same as in Experiment 1, except that the containers were lifted up by strings without any human manipulations. E2 administered the same six conditions: Both, Baited only, Balanced baited, Empty only, Balanced empty, and Control.

(3) Selecting a container: see Experiment 1.

Each dog received two trials per condition (one left and one right baiting) in a single session (12 trials in all). The order of the conditions and reward positions (left versus right) were predetermined and semirandom with the restriction that the ball did not appear more than two times in succession on the same side.

The whole session was videotaped and the choice behaviour of the subject was analysed later. Data collection and analysis methods were basically the same as in Experiment 1.

Similarly to Experiment 1, in principle, subjects could choose between the two possible target places on the basis of several simultaneous decision-making processes which can be formulated into a series of 'choice rules' (see Table 1) except for those rules which relied on the human cues ('Preferring the container manipulated by the humans' and the 'Preferring the container manipulated last') which were irrelevant in this experiment because the containers was moved by strings without any visible human manipulation.

The explanatory value of each rule in each condition for each dog was defined as in Experiment 1. Using the same categorization we analysed whether subjects relied on any of the choice rules based upon their choice or showed explicit ignorance of that rule.

\section{Results}

\section{Comparing the number of correct choices} between the testing conditions

The performance of the dogs was strongly influenced by the types of the test trials $\left(\chi_{(5,30)}^{2}=90.353, P<0.0001\right.$, Fig. 3).

The number of correct choices was at chance level in the Control condition $(T(-)=-277.5, N=30, P>0.999)$. Performance of the dogs was significantly above chance level when they had been provided direct visual information about the location of the toy (Both and Baited only conditions: $T(-)=0, N=30, P<0.0001$ in each; Balanced baited condition: $T(-)=-29, N=30, P<0.0001$ ) and also when they were shown the content of only the empty container, although both containers had been

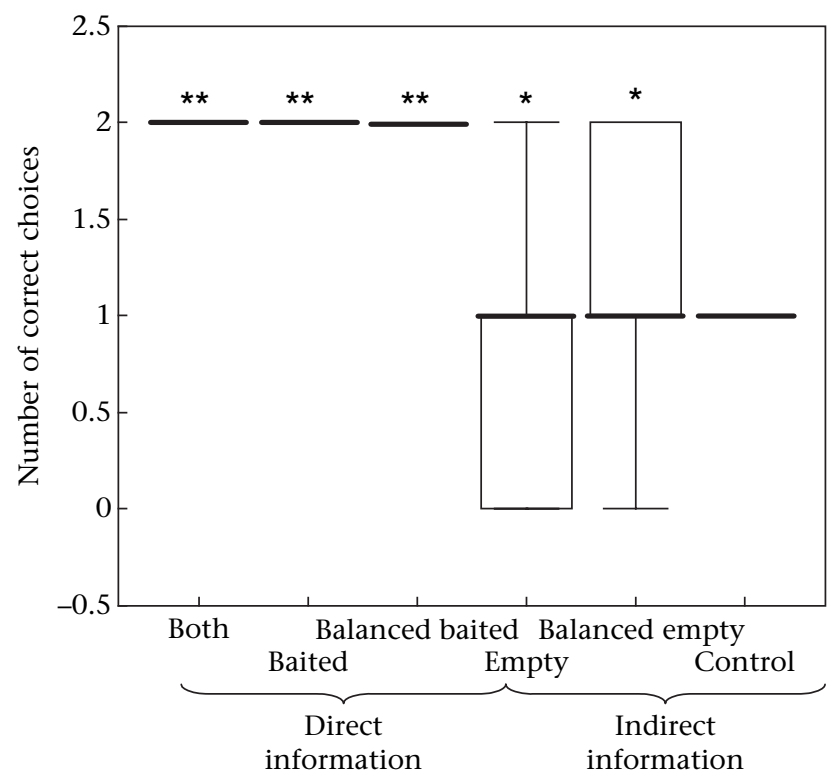

Figure 3. The number of correct choices in the different types of test trials in the 'asocial' task (Experiment 2): median, quartiles and extremes. Significant differences from control are indicated by ${ }^{*} P<0.05,{ }^{* *} P<0.01(N=35$, Wilcoxon signed-ranks tests with FDRbl adjustment).

lifted up by the strings (Balanced empty: $T(-)=-7$, $N=30, P=0.0046)$. In these three conditions the number of correct choices was significantly above the performance level shown in the Control trials (Both condition: $T(-)=-10, N=30, P<0.0001$; Baited only condition: $T(-)=0, P<0.0001$; Balanced baited condition: $T(-)=$ $-20, \quad N=30, \quad P<0.0001$; Balanced empty condition: $T(-)=-36.0, N=30, P<0.05)$.

However, when the content of only the empty container was shown (Empty condition), the dogs chose the baited one below chance level $(T(+)=8.5, N=30$, $P<0.001)$ and their performance was significantly lower compared with that shown in the Control condition $(T(+)=21, N=30, P<0.05)$.

\section{Testing the relevance of the choice rules for the description of the dogs' performance}

Table 1 shows the dogs' reliance on the predetermined choice rules in each condition and summarizes the results of the statistical analyses. As in Experiment 1, in Both condition dogs relied on their choices of 'Deductive inferring' and 'Using direct visual information' $(T(-)=0, N=30$, $P<0.01$ for both) rules. Again more important, in this condition the possible effects of the above two rules cannot be distinguished because both of them suggest the same choice (i.e. the baited container).

In the Baited only condition the dogs' behaviour corresponded significantly more strongly with the choices suggested by the 'Using direct visual information' rule and the 'Preferring the moving container' rule (comparison with chance performance: $T(-)=0, N=30, P<0.01)$. In this case, we could not decide whether dogs preferred to rely on direct visual information or on the moving of 
the baited container because the aforementioned two rules had the same outcome (i.e. only the baited container was lifted up by its strings). In the Balanced baited condition the dogs' behaviour corresponded significantly with the 'Using direct visual information' rule $(T(-)=-13.5$, $N=30, P<0.01)$.

In those trials when only the content of the empty container was presented for the subjects (Empty only condition), dogs seemingly did not use the 'Deductive inferring' rule (their choice behaviour fits this rule significantly less than what is expected by chance; $T(-)=-112, N=30, P<0.01)$, while they show a significant willingness to make decisions as predicted by the rule 'Preferring the moving container' $(T(-)=-27, N=30$, $P<0.05)$.

In contrast to the Empty only trials, in the Balanced empty condition (the content of the empty container was shown and in the case of the baited container, only the outer part was lifted up by the strings), dogs' choice behaviour could be explained by the 'Deductive inferring' rule $(T(-)=-6.5, N=30, P<0.01)$. However, they rejected to follow the 'Preferring the container moved last' rule $(T(-)=-126, N=30, P<0.05)$.

In Control trials, that is, when both containers were moved by their strings, but there was no relevant information given about the location of the bait, dogs did not rely on the 'Preferring the container moved last' rule $(T(-)=-44, N=30, P=\mathrm{NS})$, while they seemed to reject 'Preferring the previously rewarded container' $(T(-)=-90, N=30, P<0.05)$.

It is important to note that the 'Preferring the container rewarded previously' rule was not preferred in any of the conditions but even more it was significantly rejected in many cases (Both: $T(+)=31.5, N=30, P<0.01$; Baited only: $T(+)=12, \quad N=30, \quad P<0.01 ; \quad$ Balanced baited: $T(-)=-178.5, \quad N=30, \quad P<0.01 ; \quad$ Empty: $T(-)=49$, $N=30, P=\mathrm{NS}$; Balanced empty: $T(-)=-93.5, N=30$, $P=$ NS; Control: $T(-)=-90, N=30, P<0.01)$.

\section{Discussion}

Compared with the results of the first experiment, dogs showed basically the same choice preference in the different conditions. When the human social-communicative cues were excluded and the content of the empty container was revealed by means of strings, the vertical moving of the empty container (as a salient cue) per se attracted the dogs based upon their choices the same way as in the case of social marking.

In summary, the above two experiments have shown that in accordance with earlier results (Szetei et al. 2003), the sight of the reward upon cueing had the strongest influence on the dogs' choice behaviour and the effect of the movement and the 'social marking' (i.e. preferring the container manipulated by the human) also had a major impact on subjects' performance.

Interestingly, dogs did not show preference for such simple local rules as 'Preferring the container manipulated last by the human', 'Preferring the container moved last' or 'Preferring the container rewarded in the previous trial' even in the control condition when neither direct nor indirect informing cues were given.

We should note, however, that there was no such condition in which the 'Using direct visual information', 'Preferring the container manipulated by the human' or 'Preferring the moving container' rule would predict contradictory choice. So the behaviour-influencing effect of these factors cannot be compared directly.

\section{EXPERIMENT 3}

The results above have shown that human manipulation (social-communicative component: approach, gaze alternations, directional gestures towards the container) and/ or the movements of the containers (asocial-discriminative component) make that location attractive for the dogs despite the fact that the manipulated container is shown to be empty. Therefore, a third experiment was designed to answer the following questions:

Does human manipulation of the empty container override the effect of direct visual information (sight of the reward in the nonmanipulated container)?

Which component, the social-communicative or the asocial-discriminative one, can be regarded as the more important factor in the dogs' choice?

Using a novel apparatus (see Fig. 4) we could separate the following factors from one another upon cueing: (1) social cues (approach, touching, gaze alternation); (2) the movements of the container; and (3) showing the content of the container.

\section{Methods}

\section{Subjects}

Twenty-six adult pet dogs participated in this experiment and the only criterion for selection was that the dogs had to be highly motivated to play with a toy. None of them participated in the first and the second experiments.

One individual was excluded after the first few trials due to motivation problems.

The other 25 dogs (15 males, 10 females; mean age $\pm \mathrm{SD}=3.2 \pm 1.92$ years) included eight different recognized breeds (5 Border Collies, 3 Parson Russell Terriers, 1 Airdale Terrier, 2 Malinois, 1 Jagd Terrier, 1 Golden Retriever, 1 Groenendael, 1 Jack Russel Terrier, 1 Cocker

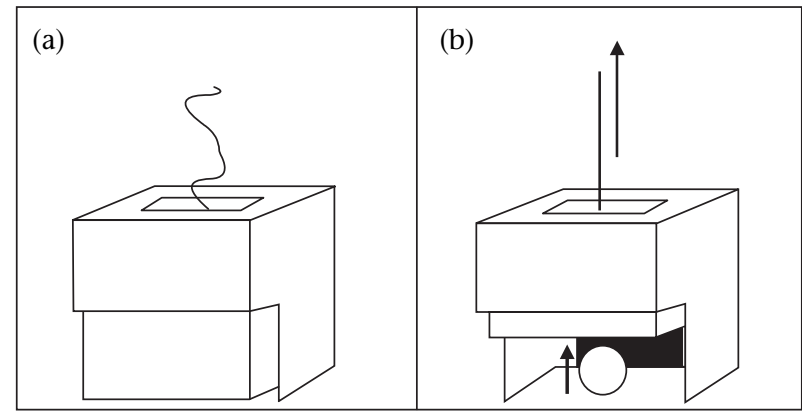

Figure 4. The container in (a) 'closed' and (b) 'open' positions. 
spaniel, 1 Husky, 1 Australian Shepherd, 1 American Bulldog, 1 Welsh Terrier) and five mongrels.

All owners were unaware of the hypotheses and goals of the study.

\section{Experimental arrangement}

Experimental observations were recorded in the same room as in Experiment 1. In this case, however, the experimenter could show the content of the boxes without any direct manipulation by hand (control was achieved remotely by a thin nylon string: see Figs 4,5 ).

The two boxes were attached to a wooden frame with two pairs of strings which were used for moving the boxes either vertically or horizontally (Figs 4,5 ). The upper side of the frame was covered by a black screen so that the experimenter could lift the front side of the boxes or could move the boxes horizontally by using the strings in an unobtrusive manner. Each box could be positioned either at the point labelled P1 (close to the frame position) or at P2 (distant position). At the beginning of the trials the dog was held by its owner at a distance of $2 \mathrm{~m}$ from the experimenter while the experimenter was standing behind the screen equidistant from the boxes and was facing the dog.

\section{Procedure}

Pretest trials. The procedure for the pretest trials was basically the same as in Experiment 1 except that the experimenter made the ball visible by lifting the inner box by the string. In the four consecutive trials all dogs except one individual met the criteria of at least three correct choices.

Test. Test trials consisted of the same steps as in Experiment 1 (Baiting, Providing information, Selecting a container) and the basic procedure was identical to that described above. After baiting, the experimenter called the dog by its name and administered one of the following conditions.

String/baited only: Boxes were placed near to the frame (P1, see Fig. 4). The experimenter stood behind the screen seemingly motionless and elevated the inner part of the baited box to reveal the ball by using the string (R1 or L1). The experimenter was wearing black sunglasses to avoid any unconscious cueing. After $3 \mathrm{~s}$ the inner box was lowered and the dog was allowed to make a choice.

String/both: Arrangement of the boxes and the way of informing the dog about the location of the reward was the same as in the 'String/Baited Only' condition; however, the experimenter showed the contents of both containers by using the strings (R1 and L1).

Human/baited only: Boxes were placed near to the frame (P1), the screen was removed and the experimenter did not wear the dark sunglasses. The way of informing was identical to the 'Baited Only' trial in Experiment 1. The experimenter stepped to the baited box and lifted its inner part, revealing the content of the box for $3 \mathrm{~s}$. In parallel she alternated her gaze three times between the dog and the manipulated container. Then she returned to

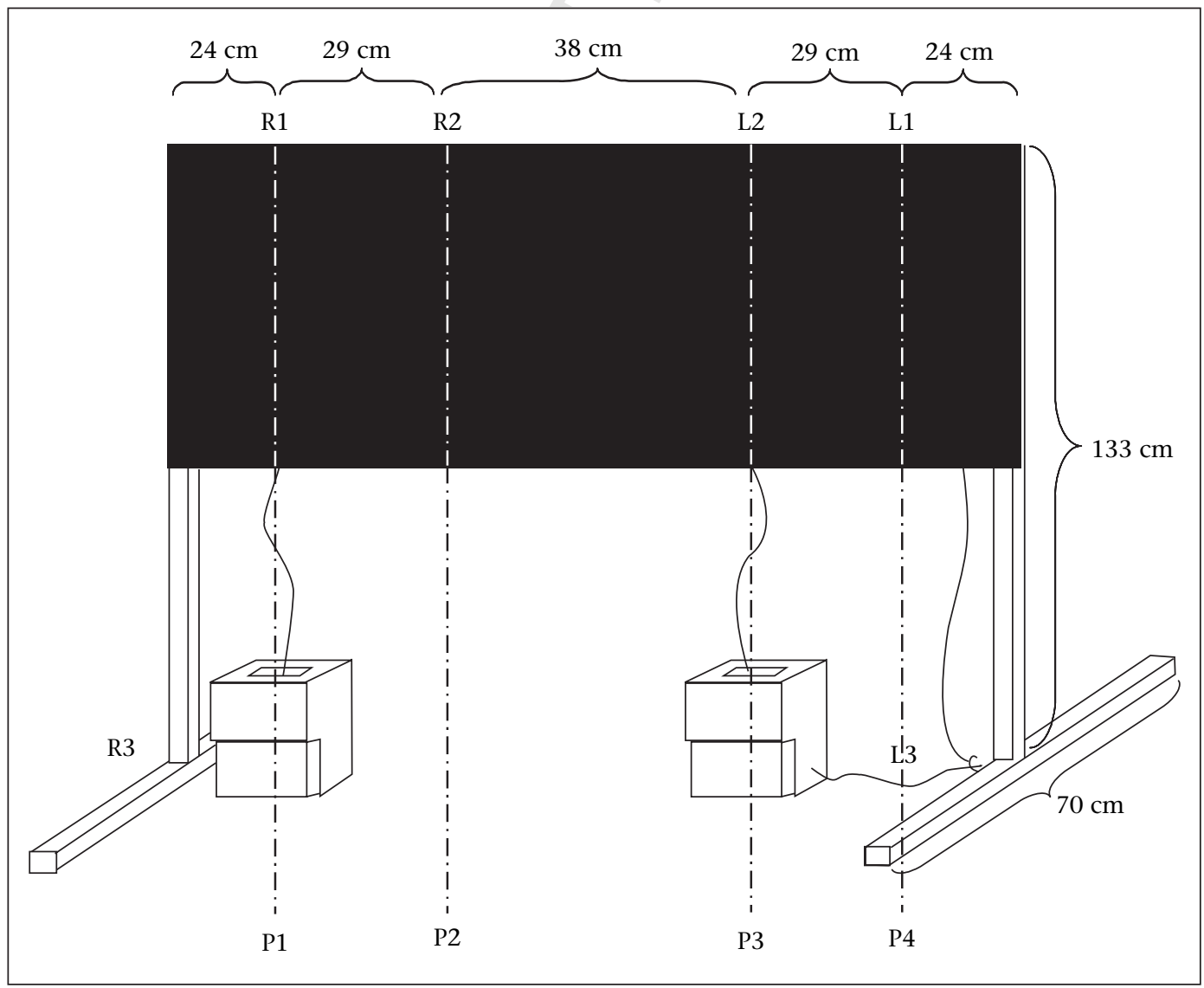

Figure 5. Experimental apparatus. There are six hooks labelled as R1-R3 and L1-L3. 
her starting position and turned her back to the dog, and the owner released the dog to make its choice.

Human/both: The position of the boxes and the method of informing was the same as in the 'Human/ Baited Only' condition with the exception that the experimenter showed the contents of both containers.

Visual cue versus human-given cues: The two boxes were close to the frame. One after the other, the experimenter showed the content of the baited box by using the string (R1 or L1) similarly as in the 'String/Baited Only' condition, and manipulated the empty box as well without showing its content: the experimenter put her palm on its top and alternated her gaze three times between the dog and the manipulated container. Finally, she returned to her starting position (behind the screen) and put her sunglasses on.

Visual cue versus box-movement: The rewarded box was placed close to the frame (P1), while the empty box was at a distant position (P2). The experimenter remained behind the screen throughout the trial wearing dark sunglasses. She showed the content of the baited box by the string (R1 or L1, as described above) and pulled the empty container from P2 to P1 position with the help of the horizontal string (R3 or L3).

Human-given cues versus box-movement: The experimenter showed the content of neither the baited box nor the empty box. One of the boxes was placed close to the frame (P1), while the other one was in distant position (P2). The experimenter put her palm on the top of the first box and alternated her gaze between the dog and this box three times. Then she returned to her starting position (behind the screen) and put her sunglasses on. The other box was remotely moved from P2 to P1 position using the horizontal string (R3 or L3). In one of these two trials the human-manipulated box was baited, and in the other trial the string-moved box contained the toy.

Control: Both hiding places were located close to the frame (P1). The experimenter did not provide information about the content of the boxes: she stood motionless wearing the sunglasses behind the screen for $10 \mathrm{~s}$ and then the dog was allowed to make a choice.

Each dog received two trials with each condition in a single session (16 trials in all). Similarly to Experiment 1 , all conditions were presented in semirandom order and the position of the reward (left versus right) was also randomly determined with the restriction that the ball did not appear more than two times in succession on the same side.

\section{Data analysis}

The number of correct choices was recorded and was compared with chance level (Wilcoxon signed-ranks tests), and Wilcoxon signed-ranks tests with FDRbl adjustment was used to make pairwise comparisons between conditions.

\section{Results}

\section{Performance in conditions involving direct} visual information

Dogs were significantly above the $50 \%$ chance performance in those conditions, when they had received direct visual information about the content of either the baited box only or both the baited and the empty ones (String/ baited only: $T(-)=0, P<0.0001$; String/both: $T(-)=0$, $P<0.0001$; Human/baited only: $T(-)=0, P<0.0001$; Human/both: $T(-)=0, P<0.0001)$. Moreover, dogs performed above chance level also in the two testing conditions when the direct view of the reward and the social cues or the movement of a box were presented on conflicting sides (Visual cue versus human-given cues: $T(-)=0$, $P<0.0001$; Visual cues versus box-movement: $T(-)=0$, $P<0.0001$ ). Accordingly, in all of these conditions (where direct visual information regarding the whereabouts of the toy was given), the dogs' performance was significantly better than in the Control trials (Wilcoxon signed-ranks tests with FDRbl adjustment: $P<0.01$ in each case).

It seems evident that when the bait was directly seen under the baited box, neither the social cues (human manipulation) nor the movement of the empty box was sufficient to distract the dogs' choice.

\section{Performance in noninforming conditions}

The number of correct choices was at chance level in the Control condition where no cues were given at all $(T(+)=13.5, P=0.547)$. In this condition, dogs chose from among the boxes randomly and they did not even show any side bias (testing the number of choosing the right side against the hypothetical median of 1 did not show significant difference; $T(+)=T(-)=68, P=1)$.

In the human-given cues versus box-movement condition where dogs were also not informed about the location of the toy, they showed preference for the humanmanipulated box, that is, dogs chose the human-manipulated box more often than the moving box $(T(-)=50$, $P=0.039$ ). Fourteen out of the 25 dogs repeatedly chose the human-manipulated box, whereas only five of them chose the moving box twice and six of them chose the human-manipulated box and the moving box once each during the two trials.

\section{Discussion}

In this experiment our design made it possible to test whether dogs rely exclusively on the direct visual information they received when they search for the toy or alternatively, human social manipulation (touching the container, gaze alternations between the container and the subject) and/or the discriminative effect of the movement of the container could have an effect on their choice behaviour. Moreover, contrasting the latter two 'nondirect informing cues' we could assess the relative importance of social-communicative cues versus the attention-catching effect of the movement of the containers. In agreement with our observations in the first experiment, these results underline the dominant role of the direct visual information (sight of the toy) when dogs are allowed to see where the toy is and then select one of the two containers. Furthermore, our results suggest that social-communicative cues (approach, touching, gaze alternation) can be considered as the more important factor for the dogs' choice in comparison with asocial-discriminative 
stimulus (movement of the container). When dogs had no direct information about the location of the toy but could witness the seemingly self-propelled moving away of one of the boxes and human communication cues (tapping, looking at) towards the other one, they showed significant preference for the human-manipulated target place. That is, more individuals chose repeatedly the 'socially marked' container than the remotely moved one.

\section{GENERAL DISCUSSION}

Our results show that dogs can make inference by exclusion: they are able to find the hidden toy on the basis of indirect visual information (sight of the empty container). This reasoning, however, is manifested in their performance only in that case when choice from among the two target places can be made neither on the basis of social-communicative cues (directional gestures, attention) nor by use of any other simple discriminative stimuli (movement of a container). The special importance of human manipulation even against other (asocial) discriminative cues is supported by the results of the third study showing that dogs preferably rely on humangiven cues versus remotely controlled movements of a target place. This is one of the few studies giving experimental evidence for inferential reasoning in dogs (Gagnon \& Doré 1992, 1993; Kaminski et al. 2004), contrary to those mainly object permanence studies which reported that dogs, unlike apes, solve the single invisible task not by inferring the location of the reward but by using simple local rules (Watson et al. 2001; Collier-Baker et al. 2004, 2006).

Collier-Baker et al. (2004) repeated the experiment of Gagnon and Doré (1992) with several control tests and reported that dogs base their search behaviour on a simple adjacency rule (searching at a box adjacent to the displacement device which originally contained the desired reward) rather than on mentally reconstructing the past trajectory of an object in the invisible displacement task. These results might have been obtained because the final position of the displacement device was an unambiguous and unbalanced cue (it was placed next to only one box). These findings are partially supported by the present study showing that dogs' choice behaviour was directed by local rules ('Preferring the moving container' and 'Preferring the container manipulated by the human') when the human social-communicative cues and moving of the hiding place were presented unbalanced on only one container for the dogs (Empty condition). However, it is important to note that if the dog received indirect visual information (the sight of the empty container) while the other cues (human communication cues and the movements of the hiding places) were presented on both containers, it did not use local rules but chose by inferring the location of the reward.

According to several studies, great apes do not simply associate cues of different modalities (visual, auditory) with reward but they seem to show understanding of the causal connections between the sight of the empty hiding place (or e.g. the noiselessness of a shaken empty cup) and the virtual place of the hidden food and inferring by exclusion (Premack \& Premack 1994; de Blois et al. 1998; Call \& Carpenter 2001; Call 2004). On the other hand, apes in general show poor performance in using human communicative cues in which one individual attempts to direct the other's attention to some third entity (see Call \& Tomasello 2003 for review). Thus, it can be assumed that they are not (so much) subjected to the prevailing bias towards cues of human behaviour (Bräuer et al. 2006), though further studies are needed for the support of this prediction.

In contrast, dogs would rather use the human communicative (pointing, looking at) and behavioural cues (trying to open a box or trying to reach something) and are skilful to understand the communicative cues of complex 'triadic' situations with humans, while they fail to use the causal cues (the presence or absence of the noise, the smell or the shape of the food) to find the hidden reward in one of two boxes. In line with the socialdog, causal-ape hypothesis suggested by Bräuer et al. (2006), we found that the human cues and/or the moving of the hiding places is much more important for the dogs than the sight of the empty container. Results support the view that although movement is an attractive cue per se which can be used by many species to track prey, human-given social cues have even higher importance for the dogs. This has been most likely intensified by the process of domestication (Miklósi et al. 2004; Bräuer et al. 2006).

Accordingly, it can be speculated that the restricted manifestation of dogs' reasoning abilities is not due to the effect of domestication in the reduction of problemsolving abilities (Frank 1980) but it is being masked by bias in following social cues. This would be in line with the dependency argument made by Topál et al. (1997).

However, to go beyond mere speculation, direct comparison with wolves, Canis lupus, would be needed. First of all we need to test whether wolves are at all capable of inferential reasoning in a similar paradigm, and, assuming they are, how manifestation of this skill is influenced by certain local rules like attraction to moving and/or human-manipulated objects. Based on the low-level use of human-given cues by young wolves to find hidden food, in comparison to apes, one can hypothesize that the performance of wolves would be less restricted by human social cues than that of dogs.

At present we only know that dogs' reasoning ability is suppressed by a prevailing bias to attend movements and human manipulation. More importantly, this behaviour can be regarded as functionally analogous to that shown by human infants, though this argument will be strengthened if the above differences are found between wolves and dogs. Many argue that in some of the cognitive tasks, human infants show similar patterns of success and error, suggesting that children's competence is often hidden by bias which is a simple heuristic whose usefulness and prevalence declines with age (Mitchell 1996).

More importantly, our experimental design allowed us to evaluate the plausibility of different choice rules and to make a more sophisticated analysis of choice situations. Based upon their choices, dogs are willing to rely 
on memory traces of simple associations between the hiding place and the sight of the reward (provided that they had visual access to the content of the baited container). However, when direct visual information about the bait is missing, social marking of the target place (by the human) is of great importance. Namely, when communicative cues and 'self-propelled' movement of the containers are contrasted, 'asocial cues' had only secondary effect on dogs' choice behaviour. It seems therefore that dogs show some competence in tasks requiring inferential reasoning; however, this ability is often masked by their bias for cues of human communication. Accordingly, dogs' behaviour may be described by a set of hierarchically ordered choice rules and different combinations of these rules which can be used by the dog to make a decision in the problem situation. Using reasoning by exclusion is one of the competing possibilities, though not the dominant one, in the case of the dog.

\section{Acknowledgments}

This research has been supported by OTKA (T029705) and the Hungarian Academy of Sciences (F01031). The authors are grateful to Mrs Celeste Pongrácz and to the four anonymous referees for their helpful comments on the earlier version of the manuscript.

\section{References}

Benjamini, Y., Drai, D., Elmer, G., Kafkafi, N. \& Golani, G. 2001. Controlling the false discovery rate in behavior genetics research. Behavioural Brain Research, 125, 279-284.

Bräuer, J., Kaminski, J., Riedel, J., Call, J. \& Tomasello, M. 2006. Making inferences about the location of hidden food: social dog, causal ape. Journal of Comparative Psychology, 120, 38-47.

Call, J. 2001. Object permanence in orangutans (Pongo pygmaeus), chimpanzees (Pan troglodytes), and children (Homo sapiens). Journal of Comparative Psychology, 115, 159-171.

Call, J. 2004. Inferences about the location of food in the great apes (Pan paniscus, Pan troglodytes, Gorilla gorilla, and Pongo pygmaeus). Journal of Comparative Psychology, 118, 232-241.

Call, J. \& Carpenter, M. 2001. Do apes and children know what they have seen? Animal Cognition, 4, 207-220.

Call, J. \& Tomasello, M. 2003. Social cognition. In: Primate Psychology (Ed. by D. Maestripieri), pp. 234-253. Cambridge: Harvard University Press.

Collier-Baker, E., Davis, J. M. \& Suddendorf, T. 2004. Do dogs (Canis familiaris) understand invisible displacement? Journal of Comparative Psychology, 118, 421-433.

Collier-Baker, E., Davis, J. M., Nielsen, M. \& Suddendorf, T. 2006. Do chimpanzees (Pan troglodytes) understand invisible displacement? Animal Cognition, 9, 55-61.

de Blois, T. S., Novak, A. M. \& Bond, M. 1998. Object permanence in orangutans (Pongo pygmaeus) and squirrel monkeys (Saimiri sciureus). Journal of Comparative Psychology, 112, 137-152.

Frank, H. 1980. Evolution of canine information processing under conditions of natural and artificial selection. Zeitschrift Für Tierpsychologie, 53, 389-399.
Gagnon, S. \& Doré, F. Y. 1992. Search behaviour in various breeds of adult dogs (Canis familiaris): object permanence and olfactory cues. Journal of Comparative Psychology, 106, 56-68.

Gagnon, S. \& Doré, F. Y. 1993. Search behaviour of dogs (Canis familiaris) in invisible displacement problems. Animal Learning and Behavior, 21, 246-254.

Gibson, K. R. 1990. New perspectives on instinct and intelligence: brain size and the emergence of hierarchical mental constructional skills. In: Language and Intelligence in Monkeys and Apes (Ed. by S. T. Parker \& K. R. Gibson), pp. 97-128. Cambridge, England: Cambridge University Press.

Hare, B. \& Tomasello, M. 2005. Human-like social skills in dogs? Trends in Cognitive Sciences, 9, 439-444.

Hare, B., Brown, M., Williamson, C. \& Tomasello, M. 2002. The domestication of social cognition in dogs. Science, 298, 1634-1636.

Heyes, C. M. 1993. Anecdotes, training, trapping and triangulating Animal Behaviour, 46, 177-188.

Kaminski, J., Call, J. \& Fischer, J. 2004. Word learning in a domestic dog: evidence for 'fast mapping'. Science, 304, 1682-1683.

Köhler, W. 1925. The Mentality of Apes. London: Routledge \& Kegan Paul.

Miklósi, Á. \& Topál, J. 2005. Is there a simple recipe of how to make friends?. Commentary Trends in Cognitive Sciences, 9, 463-464.

Miklósi, Á., Topál, J. \& Csányi, V. 2004. Comparative social cognition: what can dogs teach us? Animal Behaviour, 67, 9951004.

Mitchell, P. 1996. Acquiring a conception of mind: a review of psychological research and theory. Hove: Psychology Press.

Natale, F., Antinucci, F., Spinozzi, G. \& Poti, P. 1986. Stage 6 object concept in nonhuman primate cognition: a comparison between gorilla (Gorilla gorilla) and Japanese macaque (Macaca fuscata). Journal of Comparative Psychology, 100, 335-339.

Neiworth, J. J., Steinmark, E., Basil, B. M., Wonders, R., Steely, F. \& DeHart, C. 2003. A test of object permanence in a new-world monkey species, cotton top tamarins (Sanguinus oedipus). Animal Cognition, 6, 27-37.

Osthaus, B., Lea, S. E. G. \& Slater, A. M. 2005. Dogs (Canis lupus familiaris) fail to show understanding means-end connections in a string-pulling task. Animal Cognition, 8, 37-47.

Pepperberg, I. M., Willner, M. R. \& Gravitz, L. B. 1997. Development of Piagetian object permanence in a grey parrot (Psittacus erithacus). Journal of Comparative Psychology, 111, 63-75.

Pollok, B., Prior, H. \& Güntürkün, O. 2000. Development of object permanence in food-storing magpies (Pica pica). Journal of Comparative Psychology, 114, 148-157.

Premack, D. 1995. Cause/induced motion: intention/spontaneous motion. In: Origins of the Human Brain (Ed. by J. P. Changeux \& J. Chavaillon), pp. 286-308. Oxford, England: Oxford University Press.

Premack, D. \& Premack, A. 1994. Levels of causal understanding in chimpanzees and children. Cognition, 50, 347-362.

Soproni, K., Miklósi, Á., Topál, J. \& Csányi, V. 2001. Comprehension of human communicative signs in pet dogs. Journal of Comparative Psychology, 115, 122-126.

Szetei, V., Miklósi, Á., Topál, J. \& Csányi, V. 2003. When dogs seem to lose their nose: an investigation on the use of visual and olfactory cues in communicative context between dog and owner. Applied Animal Behavior Sciences, 83, 141-152.

Tomasello, M. 2000. Primate cognition: introduction to the issue. Cognitive Science, 24, 351-361.

Topál, J., Miklósi, Á. \& Csányi, V. 1997. Dog-human relationship affects problem solving behavior in the dog. Anthrozoös, 10, 214-224. 
Topál, J., Kubinyi, E., Gácsi, M. \& Miklósi, Á. 2006. Obeying social rules: a comparative study on dogs and humans. Journal of Cultural and Evolutionary Psychology, 3, 213-237.

Virányi, Zs., Topál, J., Gácsi, M., Miklósi, Á. \& Csányi, V. 2004. Dogs respond appropriately to cues of humans' attentional focus. Behavioural Processes, 66, 161-172.

Watson, J. S., Gergely, Gy., Topál, J., Gácsi, M., Sárközi, Zs. \& Csányi, V. 2001. Distinguishing logic versus association in the solution of an invisible displacement task by children and dogs: using negation of disjunction. Journal of Comparative Psychology, 115, 219-226.

Wood, S., Moriarty, K. M., Gardner, B. T. \& Gardner, R. A. 1980. Object permanence in child and chimpanzee. Animal Learning and Behavior, 8, 3-9.

Wright, B. C. 2001. Reconceptualizing the transitive inference ability: a framework for existing and future research. Developmental Review, 21, 375-422. 